\title{
Pollution Control of the Selati Tailings Storage Facility by Means of a Decision Support System - a Case Study
}

\author{
Angelique Daniell, Piet W. van Deventer \\ Unit for Environmental Science and Management, North-West University \\ Hoffman Street, Potchefstroom, South Africa \\ angelique.daniell@gmail.com; pietwvd@gmail.com
}

\section{Extended Abstract}

The Selati tailings storage facility (TSF) lies in the Limpopo Province in South Africa and originates from mining and the metallurgical processing of carbonatite and pyroxenite from the Phalaborwa Complex [1]. The Selati TSF consists of waste product resulting from the extraction of phosphates and production of phosphoric acid from carbonatite ore. A welldeveloped and integrated approach was followed to identify the specific nature of the problem domain and identify sound and viable solutions.

In 2005 vegetable farmers to the northwest of the TSF began experiencing a decline in both yield and quality, and increased seedling losses. Complications with the TSF's existing rehabilitation plan were identified as the resulting cause. Prevailing winds in the area originate from the southeast and result in tailings material dust being blown onto adjacent farmlands [2], which resulted in functional degradation of the land.

The Selati TSF is gap graded with $90 \%$ sand fraction and $0.9 \%$ clay fraction. The clay fraction, consisting primarily of mica, is easily transported by wind resulting dust pollution. A further factor to the dust pollution was the lack of structure and secondary clays within the TSF, which lead to the material having no linear shrinkage, and no internal cohesion. The dust transported from the TSF accumulated on adjacent farmlands, and formed crust layers, which consequently prevented the germination of seed, gas exchange and water infiltration. Furthermore dust collected on plant leaves inhibited photosynthesis, which lead to retarded plant growth and deformed vegetables. Lastly, dust generated by the TSF created abrasions on the produced crops rendering them as low grade in the trading markets.

The main objective of this study was to first ensure surface stability of the Selati TSF, with an aim to directly reduce dust generated by the TSF. To achieve this objective it was necessary to re-evaluate the existing rehabilitation plan of the mine and integrate the different rehabilitation attributes to turn the tailings materials into a medium favourable for plant growth, by addressing the following attributes:

- Both the chemical and physical deficiencies of the media

- Increase the organic matter content of media within the rooting zone by incorporation of appropriate additives such as compost

- Stabilization of the "soil" matrix on the rehabilitation site to prevent wind and water erosion and to provide a plantcover that is self-sustaining

The study leveraged a decision support system (DSS) to identify all major attributes that influenced the rehabilitation of the Selati TSF, and ultimately effectuate a semi-quantitative risk integration in support of the rehabilitation objectives.

A detailed base line assessment of the site with respect to the influence or interaction of all the attributes outlined in the DSS towards the reduction of dust pollution was implemented. Post-implementation of the DSS, it was concluded that the physical attributes of the Selati TSF had major constraints relating to rehabilitation. The rectification of said attributes has had a significantly beneficial impact on the overall functionality of the media. Gypsum from a nearby gypsum TSF was used to increase the water retention from an average of $3.83 \%$ to $12 \%$, which reduced the infiltration from $123 \mathrm{~mm} / \mathrm{h}$ to 75 $\mathrm{mm} / \mathrm{h}$. Furthermore, treatment by gypsum has increased the grading of the material and resulted in an overall decreasing in $\mathrm{pH}(\mathrm{KCl})$, from 8.84 to 8.0 within the TSF media, rendering it more suitable for the establishment of vegetation and less prone to wind erosion. 
Following a holistic approach by means of a DSS does not only provide a measurable and scientifically sound approach towards addressing the aforementioned problems arisen from pollution, while allowing for specification redefinition, but additionally actualizes a resulting solution, which is ecologically and financially viable.

Keywords: Decision Support System, Holistic Approach, Rehabilitation Attributes, Selati Tailings Storage Facility.

\section{References}

[1] W. J. Verwoerd and M. C. du Toit, "The Phalaborwa and Schiel Complex," in The Geology of South Africa, M. R. Johnson, C. R. Anhaeusser and R. J. Thomas, Eds. Johannesburg: Geological Society of South Africa, Council for Geoscience, 2006, pp. 291-299.

[2] P.W. van Deventer, Private Communication. October 2017. 